\title{
Evidence for separate pathways for color and luminance detection mechanisms
}

\author{
Kathy T. Mullen and M. Angeles Losada
}

McGill Vision Research, Department of Ophthalmology (H4.14), McGill University, 687 Pine Avenue West, Montreal H3A 1A1, Canada

Received January 13, 1994; revised manuscript received June 16, 1994; accepted June 21, 1994

\begin{abstract}
We measure threshold versus contrast (TvC) functions for chromatic (red-green) and luminance sine-wavegrating stimuli for (1) the detection of luminance in the presence of color contrast and (2) the detection of color in the presence of luminance contrast. We find that, although these crossed TvC functions both display a dipperlike shape, their facilitation differs from that found for standard uncrossed dipper functions (luminance on luminance or color on color contrast). Their facilitation disappears (cross condition 1) or is reduced (cross condition 2) by randomized presentation of the phase of the test and the mask, and the remaining facilitation (cross condition 2) displays no spatial tuning. We argue that these crossed facilitatory interactions cannot be explained by detection mechanisms with common inputs from color and luminance contrast (a nonindependence of transduction), and we present evidence that instead they reflect the use of local cues in the stimuli. We also measure the luminance-luminance TvC function in the presence of a fixed suprathreshold color contrast. The results demonstrate that, even when the color contrast produces a masking of the luminance thresholds, luminance-luminance facilitation still occurs. Thus the opposing effects of masking and facilitation can occur simultaneously. Furthermore, while luminance-luminance facilitation occurs independently of color contrast, masking can be produced by either contrast. This suggests that masking and facilitation have different underlying origins. Similar results are found for the color detection thresholds in the presence of a luminance pedestal. We conclude that there are separate pathways for the detection of color and luminance contrast, each with no input from the other contrast. We suggest that the cross masking reflects divisive interactions between these pathways that is restricted to high contrasts.

Key words: color vision, isoluminance, spatial masking.
\end{abstract}

\section{INTRODUCTION}

In the early stages of postreceptoral visual processing, spectral variations in the visual scene are extracted by cone opponent mechanisms, whereas intensity variations are extracted by cone additive mechanisms. At threshold, the number of postreceptoral detection mechanisms appears to be small; two cone opponent mechanisms and one additive mechanism are sufficient to account for the variation in detection threshold over all subtractive and additive combinations of cone catches. ${ }^{1-9}$ Evidence for a restricted set of mechanisms at detection threshold also comes from the limited number of chromatic sensations they are found at threshold. ${ }^{10,11}$

The issue of how these different detection mechanisms may be organized at a cortical level remains controversial, and different types of psychophysical model can be postulated. At one extreme, color may be processed only in combination with luminance contrast. This model, illustrated in Fig. 1(a), postulates a common pathway in which color and luminance responses share a single transduction process. It may be termed a double-duty model, since it supposes an extension of the double duty of $\mathrm{P}$ cells to the pathways of the visual cortex. The psychophysical literature, however, demonstrates little support for this model. The squared-off shape of threshold contours in a cone contrast space or similar color space suggests little summation to threshold between different postreceptoral mechanisms. ${ }^{4-7}$ Furthermore, there is little evidence for subthreshold summation between stimuli that selectively stimulate color and luminance mechanisms. ${ }^{9,12,13}$ This argues against an additive common pathway's determin- ing detection for both the cone opponent and the cone additive mechanisms. However, one contrary result, that of complete summation, has also been reported. ${ }^{14}$

Counter to the double-duty model is the idea that there are different pathways for the encoding of color and luminance contrast. Distinct color and luminance pathways, however, may still display interactions, and different types of interactive model can be proposed (see also Ref. 12). Interactions may be based on a cross sensitivity, in which the color or the luminance pathway has some degree of response to the other contrast [Fig. 1(b)]. Thus each pathway receives combined, but differentially weighted, inputs from color and luminance contrast.

Alternatively, the color and luminance pathways may be separable. We have defined a separability model, illustrated in Fig. 1(c), as one in which the different color and luminance pathways have independent transduction processes, with no residual response elicited from the other contrast. These pathways may remain independent, or they may display modulatory interaction arising after the transduction process. Figure 1(c) illustrates interactions based on mutual inhibition. It is worth noting that the models postulating distinct color and luminance pathways [Figs. 1(b) and 1(c)] do not exclude the existence of additional color-luminance mechanisms that are sensitive to combinations of color and luminance contrast. Rather, they address the relationship of the psychophysical mechanisms that determine the detection of color and luminance contrast.

The existence of the distinct chromatic and luminance pathways used in the models shown in Figs. 1(b) and 1(c) is suggested from a number of psychophysical studies in 
(a)

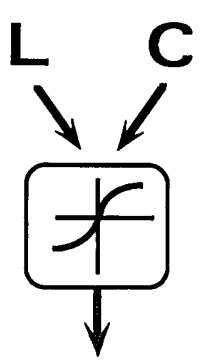

(b)

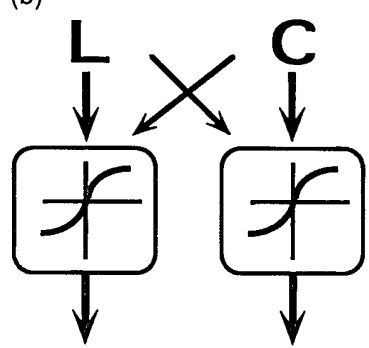

(c)

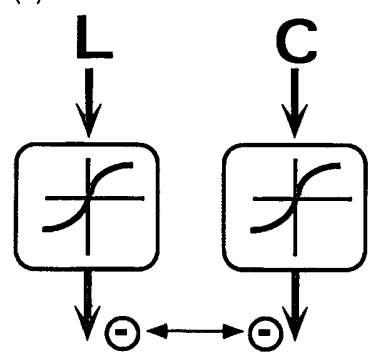

Fig. 1. Three schemes for how the color and luminance psychophysical detection mechanisms may be organized within the visual cortex: (a) double-duty model representing single common pathway for color and luminance responses, (b) distinct pathways for color and luminance mechanisms but with some crossing of the color contrast and luminance contrast inputs, (c) distinct pathways for color and luminance mechanisms with independent transduction, with modulatory interactions occurring posttransduction. L, luminance; $\mathrm{C}$, color. See text for further details.

addition to the threshold summation results already mentioned. Independent color and luminance pathways can be argued from the virtual lack of cross adaptation between color-only and luminance-only stimuli ${ }^{15,16}$ (but see Ref. 17). Independence has also been suggested by the lack of cross masking between color and luminance when broadband noise masks are used, ${ }^{18}$ although these masks may not contain sufficient contrast energy in the relevant spatial bandwidth to reveal high-contrast interactions.

On the other hand, the different masking studies that employed gratings or spots suggest that there are suprathreshold interactions between the color and luminance pathways. The forms of the results, however, are conflicting. Switkes et al. ${ }^{12}$ and De Valois and Switkes, ${ }^{19}$ using a simultaneous spatial masking technique with sine-wave stimuli, reported interactions between color and luminance contrast that are asymmetrical and in which (1) the detection of luminance in the presence of color was characterized by the absence of facilitation and robust masking, whereas (2) the detection of color in the presence of luminance showed facilitation with no masking. Different interactive models were used to account for these two conditions. On the other hand, experiments in which spot stimuli were used ${ }^{13}$ have found similar threshold-versus-contrast ( $\mathrm{TvC}$ ) functions for the detection of luminance spots on color pedestals and color spots on luminance pedestals; both functions show little subthreshold facilitation and marked suprathreshold facilitation. Masking, however, could not be measured effectively with the spot-and-pedestal stimulus arrangement. Despite the apparently interactive nature of these results, Cole et al. ${ }^{13}$ argued that the chromatic and luminance transduction processes are independent. This argument is based on the evidence that, at least for the color detection task, the facilitation of a test spot can be elicited by a range of different pedestal stimuli, such as the demarcation of the test spot area by smaller spots or lines. ${ }^{13,20}$ Such effects are hard to reconcile with explanations involving a common transduction process for color and luminance contrast.

Our aims in this paper are to assess the degree of independence of the color and luminance mechanisms and to determine the applicability of the cross sensitivity versus the separability models for luminance and color detection thresholds. We also wish to reconcile why conflicting results were obtained from the studies that used the simultaneous-masking paradigm. We use a simultaneous-spatial-masking paradigm with sine-wave stimuli. In Subsection 3.A we examine the characteristics of the $\mathrm{TvC}$ functions in the two cross conditions (the detection of luminance in the presence of color contrast and the detection of color in the presence of luminance contrast). We find that the two functions are similar and that both display interactions that show suprathreshold facilitation and some masking at high contrasts. The characteristics of the crossed TvC functions, namely, the phase dependence and the spatialfrequency tuning of the suprathreshold facilitation, suggest that these TvC functions are not the same as those found for color-color or luminance-luminance masking (the uncrossed conditions). In Subsection 3.B we provide a direct test to distinguish between the cross sensitivity and the separability of the color and luminance transducer functions as models of masking. The luminance-luminance $\mathrm{TvC}$ function is measured in the presence of a suprathreshold color contrast pedestal. The results demonstrate that the color contrast pedestal does not combine additively with the luminance contrast to influence detection threshold. Likewise, the color-color $\mathrm{TvC}$ function is measured in the presence of a suprathreshold luminance contrast, and these results, too, suggest that luminance contrast provides no additive input into the color detection thresholds. This leads us to reject the model of a cross sensitivity of these pathways in which thresholds are governed by weighted combinations of luminance and color contrasts. Instead, the results suggest that the color and luminance transduction processes, at least at low to medium contrasts, are independent, following the model of Fig. 1(c). If this is the case, the interactions observed in the crossed TvC functions must arise by a different process. In Subsection 3.C we investigate the origin of the suprathreshold facilitation in the cross conditions and provide evidence that it is based on higher-order cues. These cues appear to be different in the two cross condi- 
tions, which can lead to asymmetries in the TvC functions in certain circumstances.

\section{METHODS}

\section{A. Apparatus and Calibration}

Stimuli were horizontal isoluminant red-green chromatic gratings or isochromatic luminance gratings. Stimuli were produced by displaying two luminance modulated gratings, each on a Joyce (DM2) display screen with white P4 phosphor. These were viewed through narrow-band interference filters (Melles Griot) with center wavelengths of 525 and $605 \mathrm{~nm}$ and full bandwidths at half-height of 21-22 nm. The two monochromatic component gratings were combined $180 \mathrm{deg}$ out of phase by a beam splitter to produce a chromatic grating, or in phase to produce a luminance grating of the same mean luminance and chromaticity. Longitudinal and transverse chromatic aberrations were corrected, ${ }^{21}$ except at the lowest spatial frequency of $0.25 \mathrm{cycle} / \mathrm{deg}$. A bite bar was used to align the subject's head. Viewing was monocular and with a natural pupil. Stimuli were centrally fixated by a small fixation spot and had a mean luminance of $22 \mathrm{~cd} \mathrm{~m}^{-2}$. Linearizing calibrations of the phosphor nonlinearities of the display monitors were made with a UDT optometer (Model S370) fitted with a photometric head (no. 265). The goodness of the fits of the linearizing lookup tables to the light output of the monitors produced a contrast error for the displayed stimuli of within $0.017 \mathrm{log}$ unit. Calibrations of the absolute luminance values of the component colors were made with a Universal spot photometer. The calibrations were checked regularly throughout the study. All stimuli were generated with a VSG2/1 waveform generator (Cambridge Research Systems, Cambridge, Mass.) with 14-bit digitalto-analog converters.

\section{B. Stimuli}

Test and mask stimuli were sine waves of the same orientation. Both were Gaussian enveloped along the axis of modulation with a half-width at $1 / e$ height of 1.5 cycles of the stimulus and were sharply truncated on the horizontal axis at a bar length of four cycles of the stimulus. If test and mask stimuli had different spatial frequencies, the spatial extent of both stimuli was determined by the spatial frequency of the test grating, and the number of cycles in the masking stimulus varied inversely with the spatial frequency of the test. To achieve test spatial frequencies of 0.25 cycle/deg, we used a Zeiss (3X) telescope to magnify the display.

The luminance profile across space of the combined red, $r$, and green, $g$, component gratings is given by

$$
\begin{aligned}
L= & M_{r}+M_{g}+M_{r}(C+\Delta C) \sin \omega x \\
& \pm M_{g}(C-\Delta C) \sin \omega x,
\end{aligned}
$$

where $M_{r}$ and $M_{g}$ are the respective mean luminances of the gratings, $C$ is their contrast, $\Delta C$ is an added contrast increment or decrement, and $\omega$ is the spatial frequency. Component modulations are added to produce a homochromatic luminance grating and are subtracted for a chromatic grating. For the homochromatic luminance grating and the isoluminant chromatic grating, the component gratings have equal contrast, $C$, and $\Delta C=0$. For determination of isoluminance $\left(M_{r}=M_{g}\right)$, the measured mean luminances of the two component gratings are varied while their contrasts are held constant. Thus at isoluminance the two component gratings have identical luminance profiles, and only the color of the combined stimulus is modulated. The mean luminances remain fixed throughout the experiment. The contrasts of both the isoluminant chromatic grating and the homochromatic luminance grating are defined as the contrast of the component gratings $C$. To produce gratings with combined color and luminance contrast, the contrast of one component grating is increased and the other decreased by a fixed amount $\Delta C$. Thus if $C$ is the contrast of the original isoluminant stimulus, $\Delta C$ is the contrast of the added luminance stimulus, and vice versa.

Isoluminance of the two colors was measured with a minimum-motion method. Subjects viewed a continuously drifting grating $(2 \mathrm{~Hz})$ of 1 cycle/deg in the Gaussian window used above. We used a method of adjustment to vary the ratio of the red-to-green mean luminances in the stimulus to determine the point at which the perceived drift rate reached a minimum. This was repeated at least ten times, and an average was obtained. This is a convenient method of obtaining the isoluminant point, as there is a sharply defined minimum in perceived drift rate at isoluminance ${ }^{22-24}$ that corresponds to a null on the subject's $V(\lambda)$ luminous-efficiency function. ${ }^{22}$

A diagram of the stimuli in cone contrast space is given in Fig. 2. Only the long-wavelength-sensitive (L-) and middle-wavelength-sensitive (M-) cone plane is shown, because we use narrow-band chromatic stimuli that produce little excitation of the short-wavelength-sensitive (S) cones. Both visual mechanisms and stimuli are represented as vectors in the cone contrast space. It is important to note the positions of the stimulus vectors in relation to the vectors for the detection mechanisms. The chromatic mechanism is assumed to lie at $135 \mathrm{deg}$ $(-0.71,0.71)$, representing a cone opponent mechanism with equal L- and M-cone weightings. This assumption

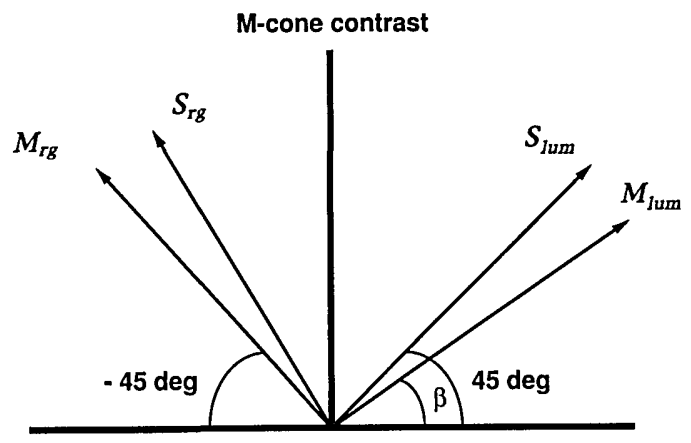

L-cone contrast

Fig. 2. Diagram of stimuli in relation to detection mechanisms in an L- and M-cone contrast space. Vectors $\mathbf{S}_{\text {lum }}$ and $\mathbf{S}_{\mathrm{rg}}$, homochromatic luminance and isoluminant red-green stimuli, respectively; vectors $\mathbf{M}_{1 \mathrm{um}}$ and $\mathbf{M}_{\mathrm{rg}}$, directions of luminance and $\mathrm{L}-\mathrm{M}$ chromatic mechanisms, respectively; $\beta$, angle of the luminance mechanism, orthogonal to the isoluminant stimulus, which is determined for each subject. The luminance stimulus lies at $45 \mathrm{deg}$, the L-M chromatic mechanism at $135 \mathrm{deg}$ (see text). 
is based on data that show the L-M chromatic mechanism to be in this direction ${ }^{5}$ and to be remarkably stable across subjects ${ }^{8}$ and spatial conditions. ${ }^{9}$ The luminance stimulus is represented by a vector lying at 45 $\operatorname{deg}(0.71,0.71)$. This vector lies orthogonal to the chromatic mechanism and thus represents the null direction of the chromatic mechanism. Isoluminance of the chromatic stimulus is determined for each subject, and the average lies at $106 \pm 4.5 \mathrm{deg}(-0.28,0.96)$. By definition the isoluminant chromatic stimulus lies in the null direction of the subject's luminance mechanism, and thus the direction of the luminance mechanism $\beta$ deduced from the isoluminant point lies at $16 \pm 4.5 \mathrm{deg}(0.96,0.28)$.

It can be seen from Fig. 2 that the color and luminance mechanisms are not orthogonal. However, each stimulus lies orthogonal to a mechanism; the luminance stimulus, although not optimally stimulating the luminance mechanism, isolates it from the color mechanism. Likewise, the color stimulus, although not optimally stimulating the color mechanism, isolates it from the luminance mechanism. This ensures that each stimulus uniquely activates one mechanism and that the data are not affected by cross stimulating between the color $(\mathrm{L}-\mathrm{M})$ and luminance threshold mechanisms.

There are, however, some assumptions inherent in the selection of these stimuli. First, we have assumed that the determination of the isoluminant point accurately represents the direction orthogonal to the luminance mechanism. The accuracy of the isoluminant stimulus is tested experimentally (Subsection 3.A). Second, we have assumed that the chromatic mechanism lies in the 135-deg $(-0.71,0.71)$ direction and is therefore orthogonal to the isochromatic luminance stimulus. If the chromatic direction deviates from 135 deg there will be some cross stimulation of the $\mathrm{L}-\mathrm{M}$ chromatic mechanism by the luminance stimulus. The possible effect of this cross stimulation on our results is tested in Appendix A. Last, we have assumed that the $S /(L+M)$ mechanism is not contributing to the results. Although the two component wavelengths in the stimuli excite the $\mathrm{S}$ cones very little, a postreceptoral $S /(L+M)$ mechanism may potentially be excited by the yellow luminance grating or by the isoluminant red-green grating. The amount of cross stimulation of an $S /(L+M)$ mechanism by the yellow grating is calculated in Appendix A. For those who dislike appendixes, we show that a relaxation of either of the latter two assumptions cannot account for the results that we find. For those interested in the issue, Appendix A shows the workings of these conclusions. It also highlights the inherent difficulties in the selection of cardinal stimuli when the exact locations of the underlying visual mechanisms are not well known.

\section{Paradigm}

In the masking experiments, thresholds were measured with a standard two-alternative forced-choice staircase procedure. A masking stimulus appeared in each of two time intervals and was accompanied by the test stimulus in one of the intervals. The contrast of the stimulus was ramped on and off with a temporal Gaussian envelope with a half-width at $1 / e$ of $125 \mathrm{~ms}$. The stimulus was stationary, and the phase of its presentation within the envelope was randomly varied between intervals. The phase relationship between the test stimulus and the mask stimulus is given for each experiment. Test contrast was controlled by a staircase procedure. The subject indicated by pressing a button in which interval the test stimulus appeared, and feedback was given after each trial. The staircase procedure was terminated after 12 reversals in the presented contrast, and the threshold was determined as the mean of the contrasts of the last 7 reversals. Each plotted threshold represents the mean of at least three measured thresholds. Results were obtained for two to four subjects (KTM, MAL, MJS, and FAK) with normal color vision measured on the standard tests (the Farnsworth Munsell 100-Hue Test and the City University Colour Vision Test).

\section{RESULTS}

A. Detection of Luminance in the Presence of Color Contrast and Detection of Color in the Presence of Luminance Contrast

In the first experiment we measured threshold-versuscontrast ( $\mathrm{TvC}$ ) function in the two cross conditions: the detection of luminance in the presence of color contrast and the detection of color in the presence of luminance contrast. Color and luminance stimuli were combined in a fixed phase relationship (180 deg) corresponding to the addition of the yellow bars of the luminance grating to

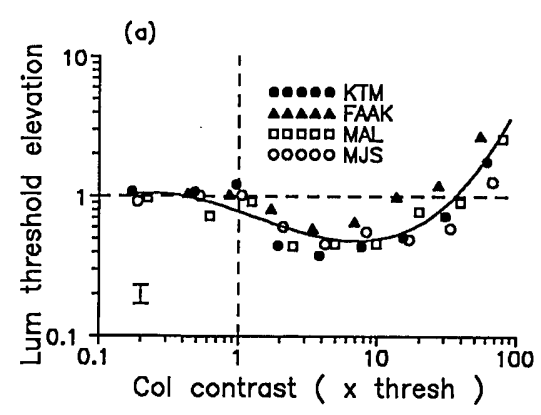

(b)

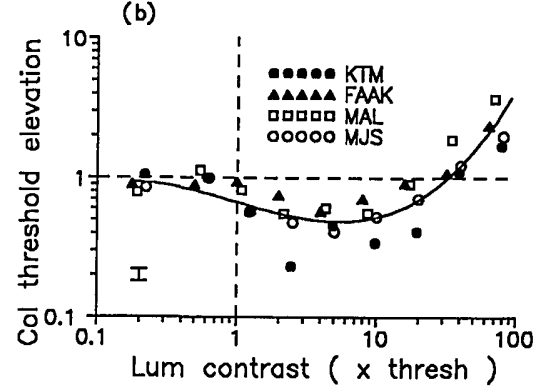

Fig. 3. (a) Detection of luminance contrast in the presence of variable color contrast. Axes are scaled in multiples of detection threshold. Stimuli are sinusoidal gratings of 1 cycle/deg; test and mask gratings are combined in a fixed relative phase of $180 \mathrm{deg}$. Solid curves, polynomial fit to the pooled data; error bars, two times the standard error of the mean. The unmasked luminance test thresholds in units of Michelson screen contrast for subjects MAL, KTM, MJS, and FAK are 0.0074, $0.0065,0.0064$, and 0.0080 , respectively. (b) Detection of color contrast in the presence of variable luminance contrast. See (a) for legend. The unmasked color test thresholds in units of Michelson screen contrast for subjects MAL, KTM, MJS, and FAK are $0.0066,0.0082,0.0075$, and 0.0092 , respectively. In this and all subsequent figures: Lum, luminance; Col, color; thresh, threshold. 
(a)
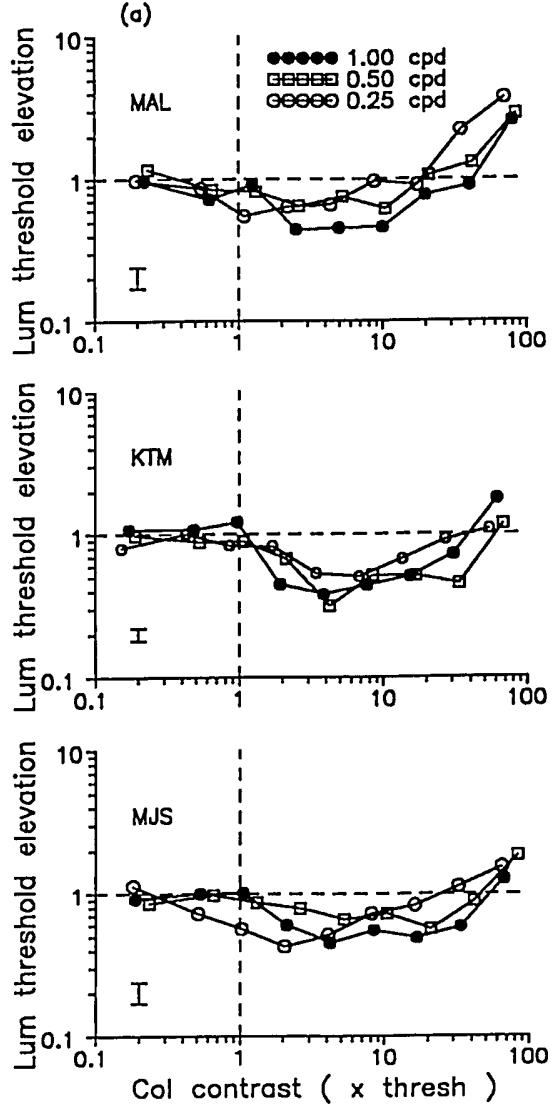

(b)
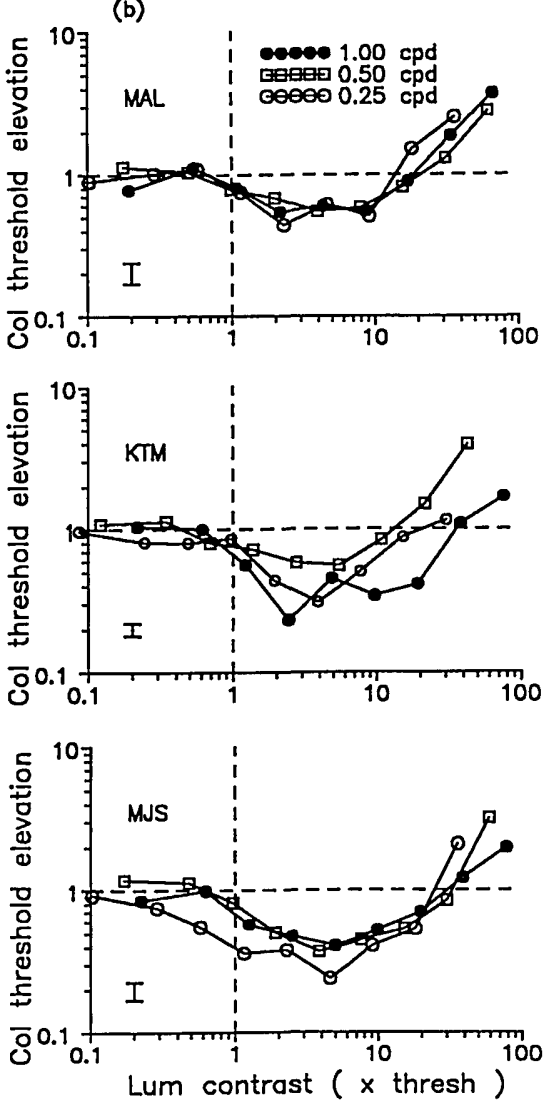

Fig 4. Crossed TvC functions for three spatial frequencies for three subjects: (a) detection of luminance in the presence of color contrast, (b) detection of color in the presence of luminance contrast. cpd, Cycles/deg. Other conditions as for Fig. 3.

the green bars of the chromatic grating. Stimuli had a spatial frequency of 1 cycle/deg. Results were obtained for four subjects and are shown in Fig. 3. In this figure (and in Figs. 4 and 5), (a) shows results for the detection of luminance in the presence of color contrast and (b) shows the detection of color in the presence of luminance contrast. The pooled results for all the subjects are fitted with a polynomial function. The TvC functions for the two cross conditions are very similar to each other and have certain characteristic features. There is no significant subthreshold facilitation. Both functions show a marked suprathreshold facilitation that is quite broad and peaks at three to eight times threshold. Both also show some masking at the higher contrasts of their respective masks (above 40 times threshold).

These functions differ from the standard dipper function found when the test and the mask are both color or both luminance contrast (termed the uncrossed conditions). Examples of the uncrossed luminance and the uncrossed color dipper functions can be seen below in Fig. 9 and 11, respectively, and are similar to those previously reported for color ${ }^{12,25}$ and luminance contrast. ${ }^{26}$ In the uncrossed TvC functions, facilitation is subthreshold, peaking near the threshold of the mask. The subthreshold facilitation is also a little narrower than the suprathreshold facilitation characteristic of the cross conditions.

There are some differences between the results for the cross conditions reported here and those from studies in which a similar sine-wave spatial masking par- adigm was used. ${ }^{12,19}$ In Fig. 3 the detection of luminance in the presence of color shows a marked facilitation (approximately twofold) compared with the absence of facilitation that was reported previously. Our results resemble those obtained with spot stimuli, ${ }^{13}$ which display suprathreshold facilitation at three to eight times threshold in both cross conditions, results that are similar to our data plotted in Fig. 3. We find that the two cross conditions display significant and similar masking. Previously, masking was reported only for the detection of luminance in the presence of color. ${ }^{12}$ However, plotting our data and those of Switkes et al. ${ }^{12}$ on the same graph reveals that there are only small differences between the two data sets in the high-contrast masking region. We conclude that there are no systematic asymmetries of masking between the two conditions.

\section{Effect of Spatial Frequency}

We extended the measurement of the crossed TvC functions to other spatial frequencies, especially to the lower spatial frequencies, for two reasons. First, if the shape of the $\mathrm{TvC}$ functions is dependent on the presence of chromatic aberration in the stimulus, both the facilitation and the masking would be expected to disappear at low spatial frequencies. Second, it has been suggested that the masking of luminance by color contrast is spatial frequency dependent, disappearing at low spatial frequencies. ${ }^{12}$ Results are shown in Fig. 4 for three spatial frequencies $(1.00,0.50$, and 0.25 cycle/deg) for three subjects. Test and mask stimuli were presented in the 

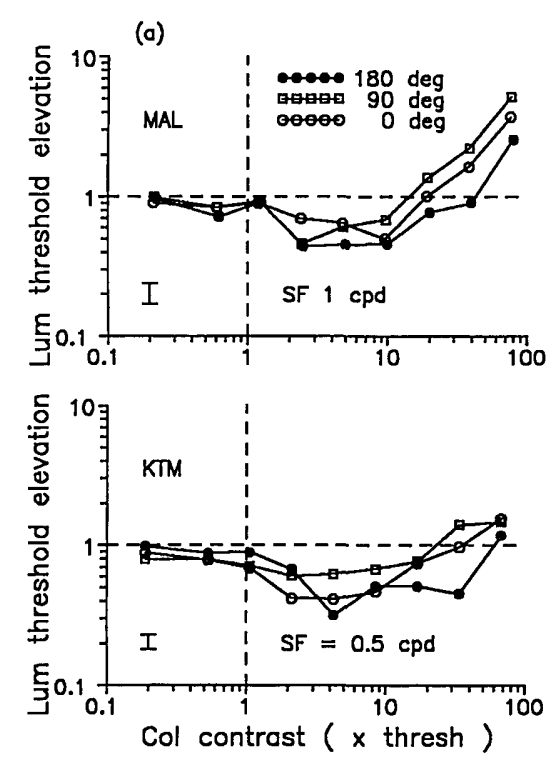

(b)
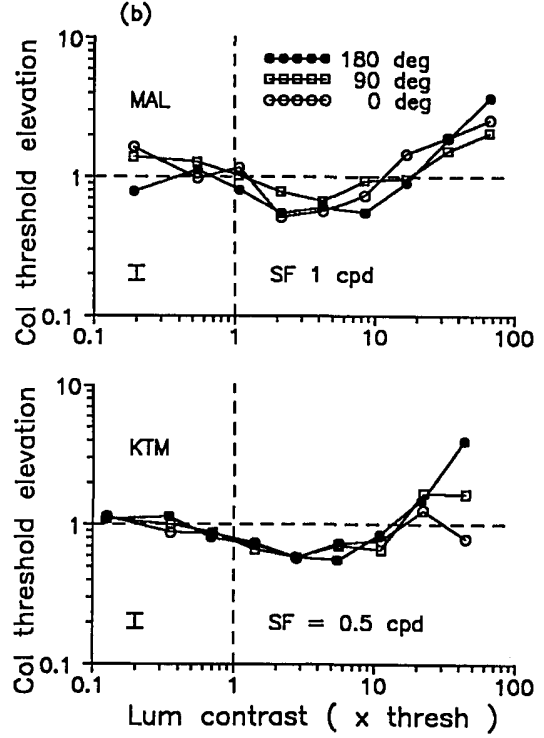

Fig. 5. Crossed TvC functions for three phases of test and mask combination for subjects MAL and KTM: (a) detection of luminance in the presence of color contrast, (b) detection of color in the presence of luminance contrast. Spatial frequencies as marked; cpd, cycles/deg. Other conditions as for Fig. 3.

same fixed phase (180 deg) as for Fig. 3. Although both facilitation and masking show some variability with spatial frequency, both remain apparent at all spatial frequencies. In particular, there is no loss of facilitation at the lowest spatial frequency (open circles) for the detection of luminance in the presence of color, indicating that chromatic aberrations are not influencing the results. In support of these results, the facilitation of color-threshold spot stimuli by luminance pedestals occurs over a range of spot sizes. ${ }^{9,13}$

\section{Effect of Fixed Phase}

In this experiment we compare $\mathrm{TvC}$ functions for three different fixed phases of combination of color and luminance contrasts $(0,90$, and $180 \mathrm{deg})$. This is a further test for the presence of spurious luminance contrast in the chromatic stimulus, which arises from chromatic aberration or from an inaccurately measured isoluminant point. The effects of chromatic aberration will be greatest for the higher color contrasts and so would particularly be expected to influence the detection of luminance in the presence of color contrast. If chromatic aberration were significant, this $\mathrm{TvC}$ function might reflect the effect of spurious luminance contrast in the chromatic mask stimulus and represent a standard luminance dipper function slid rightward along the mask contrast axis. In this case the shape of the TvC function would depend on the relative phase of the test and the mask. In the case of longitudinal chromatic aberrations or an inaccurate isoluminant point, facilitation would be expected to occur when the test contrast and the spurious luminance contrast of the mask are in phase, but it would be lost or greatly reduced when the test contrast and the mask luminance contrast are in the opposite, canceling phase (see Cole et $a l .{ }^{13}$ for examples of antiphase luminance dipper functions). Transverse chromatic aberration, by misaligning the component monochromatic gratings, might produce some luminance contrast closer to a \pm 90 -deg phase relationship with the luminance test stimulus ${ }^{27}$ and would be reduced or eliminated by the presentation of test and mask between 0 and 180 deg of relative phase.

Figure 5 shows the results for test and mask combinations at 0,90 , and 180 deg for two subjects. Thresholds for the suprathreshold facilitation and masking are scattered. However, all phases produce thresholds with clear suprathreshold facilitation, and there is no systematic variation with phase. These results indicate that spurious luminance contrast in the chromatic stimulus cannot account for the shape of the $\mathrm{TvC}$ functions in either cross condition. These results are also in keeping with those of De Valois and Switkes ${ }^{19}$ and Switkes et al., ${ }^{12}$ who report no significant phase dependence of masking for 0 -, $90-$, and 180-deg test and mask combinations. The fact that the suprathreshold facilitation in the cross conditions occurs over the full range of fixed phases distinguishes it from the subthreshold facilitation of the uncrossed conditions, which is confined to test and mask phases of $0 \mathrm{deg}$.

\section{Effect of Phase Randomization}

In this experiment, two phases of the test and mask combination ( 0 and $180 \mathrm{deg}$ ) were presented randomly on a trial-by-trial basis. The random presentation changes the local color appearance of the test-plus-mask combination from trial to trial and ensures that learning of the local color changes between the two intervals cannot be used by the subject as an aid to detect the test. For example, for the detection of luminance contrast in the presence of color, adding the luminance test in a fixed phase of 180 deg causes the red to darken (appear browner) and the green to brighten. Thus, comparing one color between the two intervals, for example, to find the browner red, may be used to indicate the luminance test interval. In the random phase condition, however, the luminance test may cause the red either to brighten or to darken, and so no local color cues to the presence of the test stimulus exist.

The results for luminance detection in the presence of color contrast are shown in Fig. 6. The facilitation disappears with random phase presentations for subjects MAL and MJS. For KTM some facilitation remains, although 

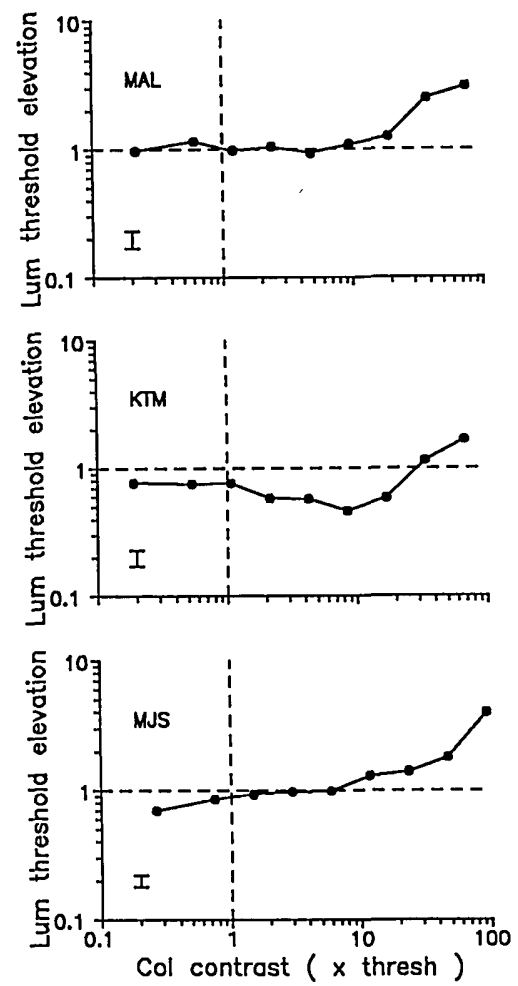

Fig. 6. Detection of luminance in the presence of color contrast when the relative phases of test and mask stimuli are randomly presented at 0 or 180 deg for three subjects. Conditions are as in Fig. 3. Test spatial frequencies are $1,0.5$, and 1 cycle/deg for subjects MAL, KTM, and MJS, respectively.

it is reduced in comparison with the fixed phase conditions. It is worth noting, that since similar thresholds were obtained in both fixed phase conditions ( 0 and 180 deg), the loss in facilitation found in this experiment is not due to the mixing of trials that have different levels of detectability. These results imply that the suprathreshold facilitation found in the fixed phase condition may be based on the use of local comparisons between the two intervals rather than on the detection of a luminance modulation across one stimulus. The nature of these cues is investigated in Subsection 3.C.

The results for the other cross condition (the detection of color in the presence of luminance) with random phase presentation are shown in Fig. 7. For all three subjects some facilitation remains when test and mask are in random phase. This implies that the facilitation in this cross condition is not likely to be accounted for by the use of local changes in color appearance.

\section{Suprathreshold Facilitation When Test and Mask Have Different Spatial Frequencies}

In this experiment we vary the spatial frequency of the test relative to the mask for each of the two cross conditions. The reason for this experiment is twofold. First, if the facilitated detection at suprathreshold mask contrast is based on a local stimulus feature in the testplus-mask combination, detection may be disrupted by the presentation of test and mask at different spatial frequencies, which alters the stimulus appearance. Second, if the TvC functions represent detection by color and luminance pathways that have some cross sensitivity, the interaction may be increased when test and mask are of different spatial frequencies. For example, a color detection pathway may have some sensitivity to luminance contrast but with different spatial passbands for color and luminance, thus displaying greater interactions when the spatial frequency of the mask is displaced from the spatial frequency of the test. ${ }^{28}$

Complete TvC functions for the detection test stimuli with masks at 1 and 2 octaves higher and lower than the test were obtained with the same paradigm as for Fig. 3-5. Results for the detection of luminance in the presence of color are shown in Fig. 8. The raw data are not shown. From each complete TvC function, however, the measure of masking was taken as the threshold at the highest mask contrast $(50 \%)$, and the minimum threshold was taken as the measure of facilitation. Data are plotted in multiples of the unmasked threshold. Two conditions for the combination of luminance test and color mask were used: a fixed phase of $180 \mathrm{deg}$ (open symbols) or 16 phases randomly presented from trial to trial (filled symbols). Results show that suprathreshold facilitation occurs only for the 180-deg phase combination and that it is tuned, disappearing when the spatial frequencies of test and mask differ by 1 or more octaves. Randomizing the test and mask phase produces a loss of facilitation. Both phase randomization and variation of the test-plus-mask spatial frequency may remove the suprathreshold facilitation by eliminating local cues in the stimuli. Masking remains when test and mask differ in spatial frequency over a range of \pm 2 octaves and displays a broad spatial-frequency selectivity. Spatially selective masking has been re-
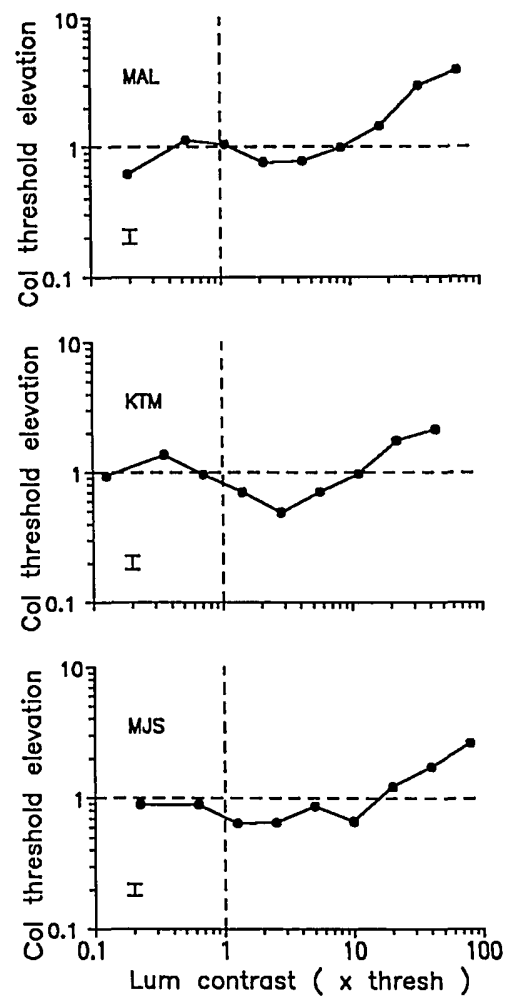

Fig. 7. Detection of color in the presence of luminance contrast when the relative phases of test and mask stimuli are randomly presented at 0 or 180 deg. Details as for Fig. 6. 

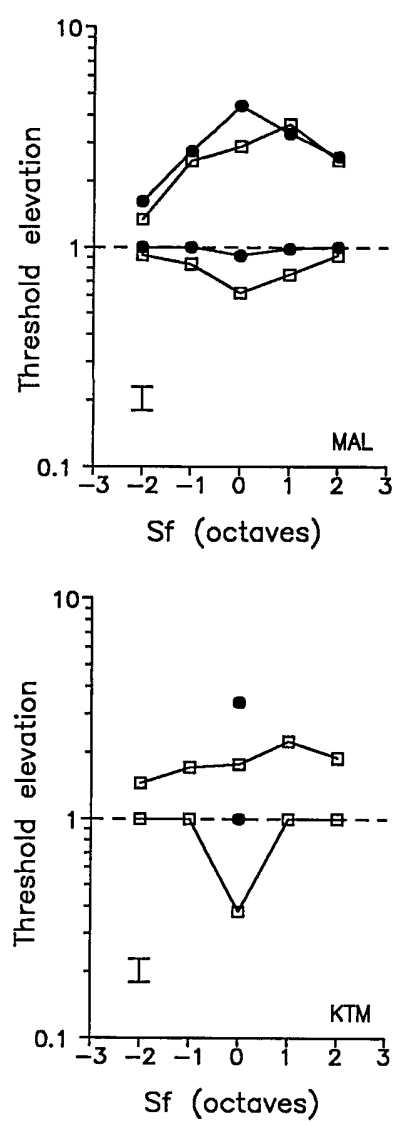

Fig. 8. Change in threshold (expressed as multiples of unmasked threshold) for the detection of a luminance test in the presence of color contrast as a function of the relative spatial frequencies, Sf, of the test and mask (in octaves) for subjects MAL and KTM. Data are derived from measurements of complete TvC functions (not shown). Masking was obtained with a fixed mask contrast $(0.50)$. Facilitation is represented by the lowest threshold of the complete TvC function. Open symbols, fixed phase of test and mask (180 deg); filled symbols, random presentation of 16 test and mask phases; error bars, two times the standard error of the mean. Spatial frequency, 0.50 and 1 cycle/deg for MAL and KTM, respectively. Note that facilitation is lost when test and mask are presented randomly and when they differ in spatial frequency.

ported for this condition by Switkes et al. ${ }^{12}$ and De Valois and Switkes. ${ }^{19}$

For the detection of color in the presence of luminance contrast, a different pattern of results is found. Results are shown in Fig. 9, with the same format as in Fig. 8. Suprathreshold facilitation is reduced but is not eliminated in the randomized phase condition. Curiously, it shows no spatial tuning but has a constant magnitude when test and mask frequencies differ by as much as 2 octaves. The lack of spatial tuning distinguishes this suprathreshold facilitation from the subthreshold facilitation found in the uncrossed conditions, which has a narrow tuning of less than 1 octave. ${ }^{12,26}$ Masking in this cross condition is again spatially selective, although the results suggest that this cross condition may have a narrower tuning than the reverse condition shown in Fig. 8. The masking in both cross conditions, although reduced in magnitude, has spatial characteristics that are broadly similar to those of the masking found in the uncrossed conditions. ${ }^{12,25}$

\section{B. Separability of Color and Luminance Response Functions}

In this section we investigate the nature of the interactions between color and luminance contrast in the two cross conditions. We distinguish between two possibilities. On the one hand, changes in test threshold may be produced by the mask because the color and luminance pathways each retain a residual sensitivity to an input from the other contrast [the cross-sensitivity model of Fig. 1(b)]. In this case the crossed TvC function would represent a standard dipper function slid rightward along the mask contrast axis. On the other hand, the transductions of color and luminance contrast may be independent but with modulatory interactions occurring at a subsequent stage [the separability model of Fig. 1(c)]. The following experiments aim to distinguish between these two hypotheses.

In the first experiment we test for separability versus additivity of the luminance response function. The paradigm is to measure a luminance dipper function (uncrossed luminance $\mathrm{TvC}$ function) in the presence of a pedestal of fixed color contrast. The color contrast of the pedestal grating is chosen to be sufficiently large to show a threshold elevation of the luminance test stimulus. The selection of the contrast of the color pedestal grating for one subject (MAL) is shown in Fig. 10(b). To this grating of fixed color contrast we add a variable lumi-
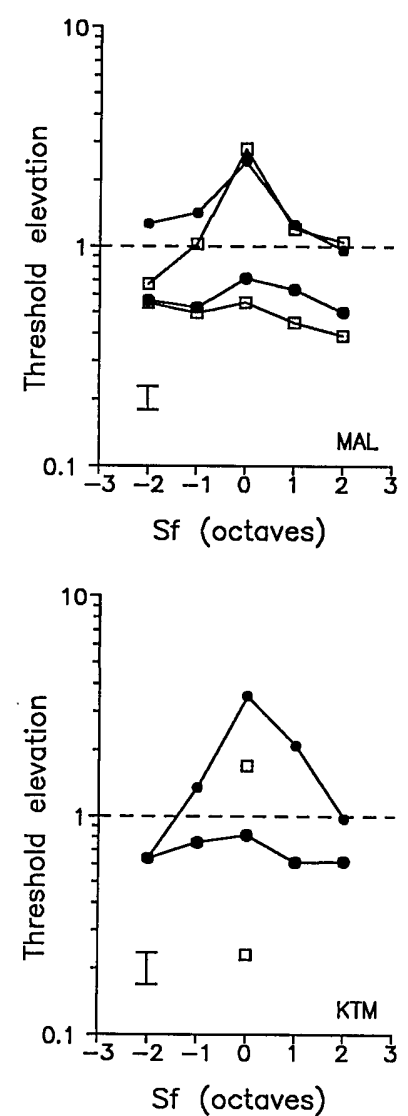

Fig. 9. Change in threshold for detection of a color test in the presence of luminance contrast as a function of the relative spatial frequency, Sf, of test and mask stimuli. Details as for Fig. 8. Note that the facilitation remains for the random presentation of test and mask even when test and mask spatial frequencies differ. 
(a)

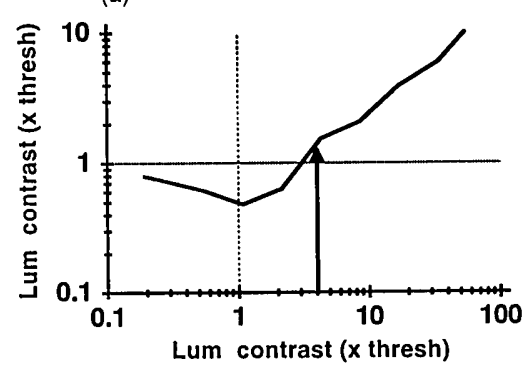

(b)

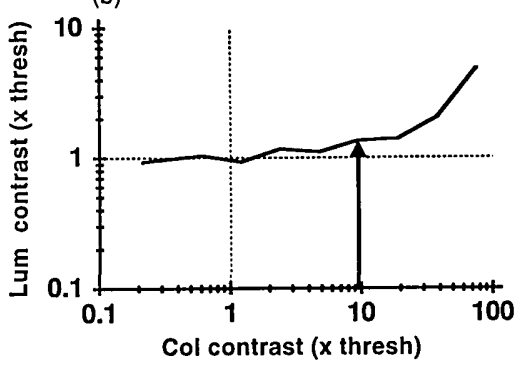

Fig. 10. Calculation of equivalent contrasts of color and luminance masks for detection of a luminance test grating for subject MAL: (a) uncrossed luminance TvC function, plotted in the standard way; (b) crossed TvC function measured with the test and mask phase randomized over 16 different phases. The arrows indicate the luminance and color mask contrasts that produce an equivalent luminance test threshold elevation. Test and mask spatial frequency is 1 cycle/deg. Data points are not shown.

nance contrast mask and measure test thresholds. The phase of the luminance test and luminance mask is fixed $(0 \mathrm{deg})$, but the phases of the luminance mask and the color pedestal are varied randomly from trial to trial. If the luminance detection mechanism receives additive weighted inputs from both the luminance and the color contrast of the mask, the luminance test threshold will continue to rise with increasing luminance contrast. If, on the other hand, the luminance response function receives no additive input from color contrast, a standard luminance dipper (TvC) function will be obtained regardless of the presence of the fixed color contrast. In this case the luminance TvC function would show separability. These predictions are formalized into two models:

1. Additivity of Color and Luminance Contrast. The first model assumes that both color and luminance contrast combine additively, with different weightings, to the luminance test threshold. The luminance contrast of the mask is known, but the effective contrast of the color mask to the luminance response mechanism is required. One obtains this by equating the luminance and color contrast masks that produce an equivalent test threshold elevation. We measured a luminance-luminance TvC function [Fig. 10(a)] and fitted the rising part of the function with a linear regression on log-log coordinates. From this we obtained the contrast of the luminance mask that produces the same luminance test threshold elevation as the color contrast mask. For subject MAL in Fig. 10, luminance contrast is two times more effective than color contrast as a mask. We predict the threshold elevation for the combined mask (fixed color contrast plus variable luminance contrast) by using these weight- ings and the linear fit of the rising section of the luminance-luminance TvC function.

2. Separability of Color and Luminance Contrast. The second model predicts the results that would be expected if the luminance response function had no additive input from the color contrast mask. We shifted the standard luminance-luminance $\mathrm{TvC}$ functions obtained for each subject [e.g., Fig. 10(a)] up and along the horizontal axis to take into account the elevated luminance test and mask threshold obtained with fixed color contrast. The separability model predicts that the variable luminance contrast mask will produce the same facilitation of the luminance test threshold in the presence of the fixed color contrast mask as is obtained for the standard luminance dipper function.

Figure 11 shows the results for three subjects. Luminance test thresholds are plotted as a function of the variable luminance mask contrast. The color contrast of the fixed pedestal is 0.06 for each subject. The leftmost symbols indicate the threshold obtained for the luminance test in the presence of the color mask, with no added luminance contrast. The solid curves give the additivity model prediction, and the dashed curves show the prediction based on separability. It is worth noting that test thresholds were measured only for low to medium mask contrasts (less than ten times threshold), because the two model predictions come together at higher mask contrasts. The data fall significantly and consistently below additivity, and the result for all three subject fail to follow the additivity prediction. A crucial finding is that all subjects show a decrease in test threshold with added luminance contrast, despite the initial threshold elevation of the test produced by the fixed chromatic pedestal. The facilitation is greatest around threshold of the luminance mask. The data are well predicted by the separability model, suggesting that the luminance response function is separable from color contrast and that luminance transduction has no input from the color contrast of the mask.

The experiment was repeated for the detection of color contrast. Figure 12 shows the calculation of the equivalent contrast of the fixed luminance contrast mask [Fig. 12(b)] from the color-color TvC function [Fig. 12(a)]. For subject KTM, color contrast was four times as effective as luminance contrast at elevating the color test threshold. The results for all three subjects are shown in Fig. 13; the same methods were used as for Fig. 12. The solid curves show the prediction for additivity. The dashed curves show the prediction for separability, representing the color-color TvC function shifted to match the test and mask threshold obtained with the fixed luminance pedestal. These results are not predicted by the additivity model. There is a decrease in the color test threshold with the added color contrast that produces a dipperlike function. However, this facilitation has a broader and flatter shape than the uncrossed facilitation (dashed curves). The separability model would be supported if threshold in the presence of the fixed luminance mask followed the same standard dipper function that is found for the uncrossed condition. Although all the results show facilitation, the facilitation differs from that in the standard dipper function. Thus the results suggest that color transduction has no additive input from lumi- 


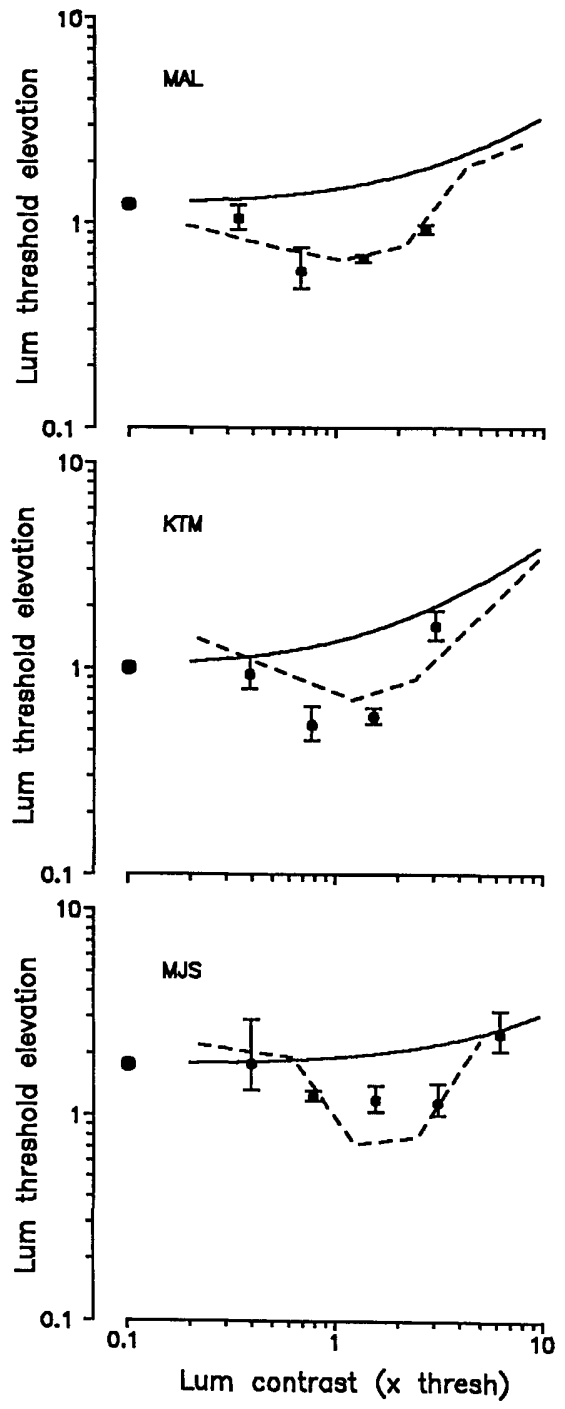

Fig. 11. Luminance test thresholds as a function of luminance mask contrast in the presence of a fixed pedestal of color contrast for three subjects. Axes are scaled in units of unmasked luminance test threshold. The fixed color contrast pedestal is selected to produce a small threshold elevation of the unmasked luminance test stimulus, as indicated by the arrow in Fig. 10(b) and corresponding to the leftmost symbol in each panel of this figure. The Michelson contrast of the color pedestal is 0.06 for each subject. The relative phase of the luminance test and the luminance mask is $0 \mathrm{deg}$. The phase of the fixed color pedestal in relation to the luminance test and mask varies randomly over 16 phases. Solid curves, prediction for an additive model for the combined influence of color and luminance contrast on the test threshold (see text); dashed curves, prediction for the separability model, which assumes that the color and luminance transduction processes are independent (see text).

nance contrast, but whether color transduction shows a true separability from luminance contrast remains to be firmly established.

\section{Evidence for the Use of a Local Color Cue in Suprathreshold Discriminations}

The results presented in Subsections 3.A and 3.B suggest that the interactions between color and luminance contrast cannot be accounted for by a cross sensitivity between color and luminance pathways. Furthermore, the finding that the phase randomization of the test and the mask effectively removes the suprathreshold facilitation for the detection of luminance contrast and reduces it for the detection of color contrast points to the role of local color cues in determining detection thresholds. In this section we investigate whether such local cues may provide a likely explanation for the suprathreshold facilitation in the two cross conditions.

\section{Detection of Luminance in the Presence of Color Contrast}

As was discussed in Subsection 3.A, the fixed phase condition potentially provides local color cues for detection of the test stimulus. For example, adding luminance contrast in a phase of $180 \mathrm{deg}$ causes the green to brighten and the red to darken in comparison with the isoluminant stimulus. Two of the subjects reported that the browning of the red bars was a useful cue to the detection of the luminance test. The third subject reported using the brightening of the green as a cue. In other words, the subject may base a response on a comparison of the color appearance of one set of bars between the two intervals rather than on an examination of each stimulus for a luminance modulation, which requires a comparison of the red and the green bars within the same stimulus. The two different strategies may produce different results.

In this experiment we test the subjects' ability to use the local color cue to improve discrimination. As above (see Fig. 6), the test-and-mask combination is randomized from trial to trail at either 0 or $180 \mathrm{deg}$ of phase. However, in this experiment the subject was specifically instructed to compare the red bar color between the two intervals and to select the grating with the darker red. Note that if the luminance test is added at 0 deg the red brightens and the green darkens, and the isoluminant interval has the darker red. If the luminance is added at

(a)

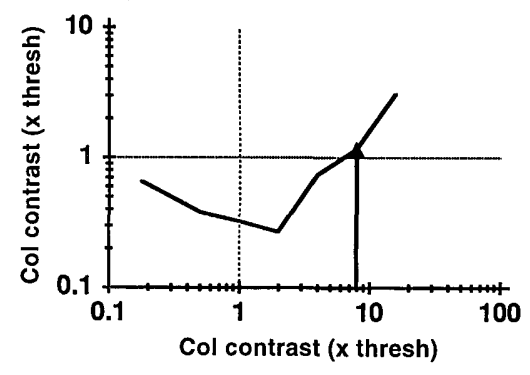

(b)

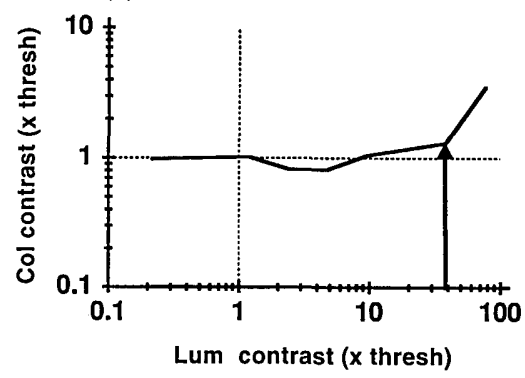

Fig. 12. Calculation of equivalent contrasts of color and luminance masks for detection of the isoluminant chromatic test grating for subject KTM. (a) Uncrossed color TvC function, plotted in the standard way; (b) crossed TvC function, measured with the test-and-mask phase randomized over 16 phases. The arrows indicate the luminance and color mask contrasts that produce an equivalent color test threshold elevation. Data points are not shown. 


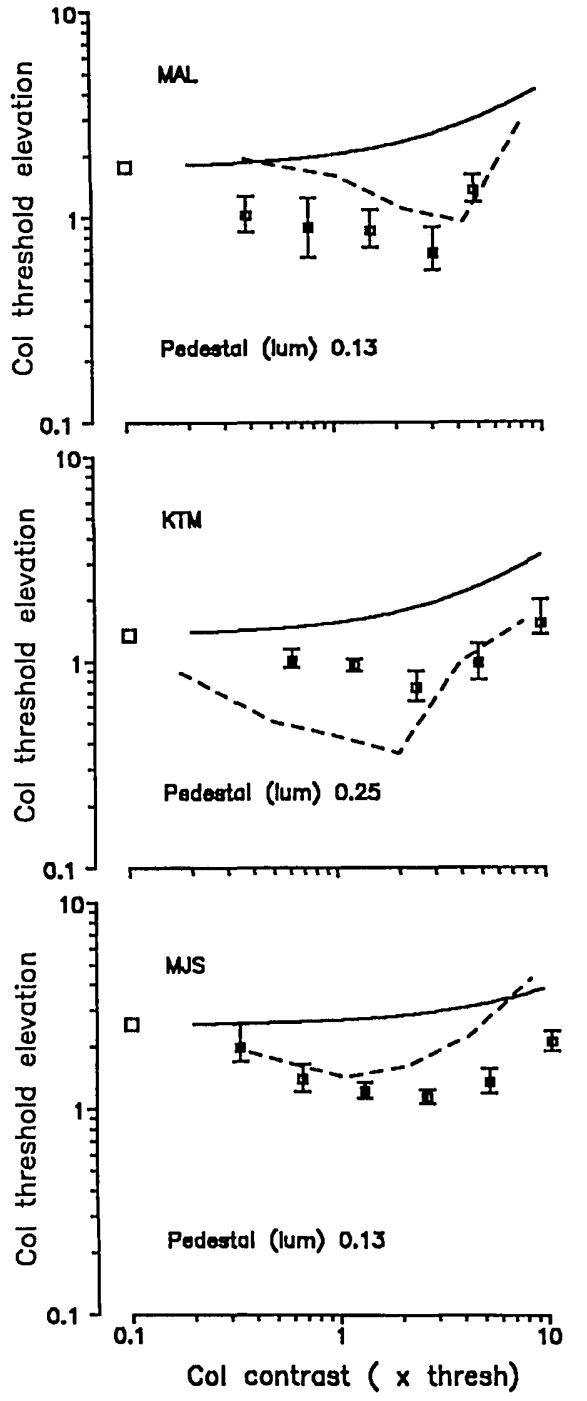

Fig. 13. Color test thresholds measured as a function of color mask contrast in the presence of a fixed pedestal of luminance contrast for three subjects. Axes are scaled in units of unmasked color test threshold. The fixed contrast of the luminance pedestal, given in each panel, is selected to produce a small threshold elevation of the unmasked luminance test stimulus, as indicated by the arrow in Fig. 12(b) and corresponding to the leftmost symbol in each panel of this figure. Remaining details as for Fig. 11.

$180 \mathrm{deg}$, the red darkens and the green brightens, and the luminance test interval has the darker red. Hence a local chromaticity discrimination must be used for correct detection, but this cue is not correlated with the presence of the luminance test stimulus. This allows its usefulness to be assessed independently from the detection of the luminance test contrast.

The results are shown in Fig. 14 by the symbols. (Since these thresholds are with a suprathreshold mask, the results fall to the right of the threshold line on the abscissa.) For comparison, the thresholds for the detection of test luminance contrast in the randomized phase condition (the results plotted in Fig. 6) are shown by the solid curves. The results show that for all three subjects the use of the local color cue significantly lowers thresholds, in comparison with the detection of the test luminance contrast in the randomized phase condition. Thus the comparison of the relative changes in the appearance of local regions of the stimulus in the two-alternativeforced-choice experiment appears to contribute to the suprathreshold facilitation.

We have used the term local color cue to refer to the change in chromaticity that occurs between the peaks of the red bar in the isoluminant grating and in the grating with the added luminance contrast. A chromaticity change will also occur between the peaks of the green bars. These cues reflect the changes in proportions of red and green lights that occur when the yellow luminance increments or decrements are added to the red or the green bars of the isoluminant stimulus. Although both sinusoidal luminance modulation and chromatic modulation have approximately the same mean chromaticities, adding the (yellow) luminance grating to the chromatic grating alters the relative proportions of red and green, especially at the peaks and troughs. This change reflects the fact that the chromaticity profile, if considered to be the modulation in the proportions of red and green lights, becomes nonsinusoidal in the presence of the luminance contrast $^{29}$ It is also possible that a local color cue may enhance sensitivity in ways other than by permitting a direct chromaticity discrimination, for example, by reducing uncertainty about the location of key stimulus features.

Detection of Color in the Presence of Luminance Contrast We applied the same experiment to the detection of color in the presence of luminance contrast. If the stimuli are combined in a relative phase of $0 \mathrm{deg}$, the yellow bars of
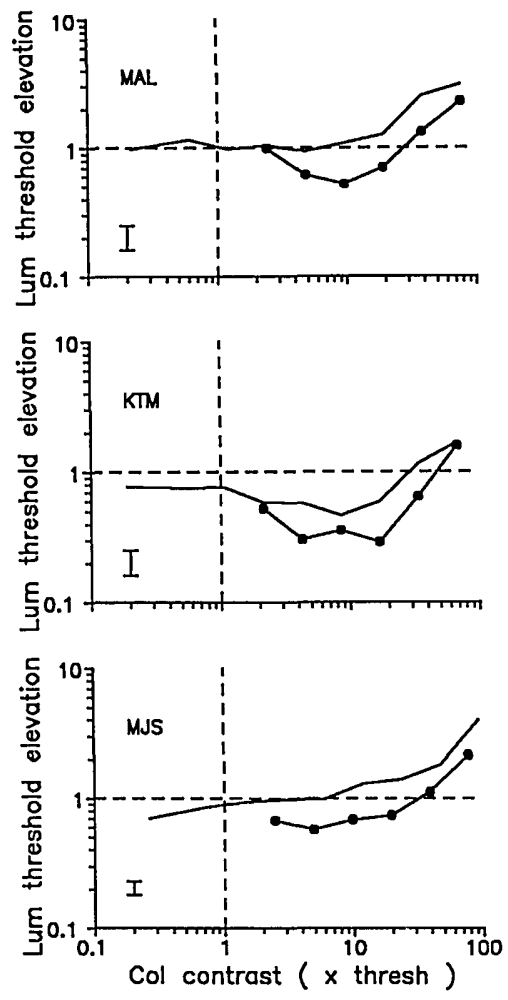

Fig. 14. Luminance test thresholds as a function of color contrast for three subjects. Filled symbols, thresholds for the detection of a local color cue based on a comparison of the stimuli in the two presentation intervals (see text). Curves without symbols, random presentation of test and mask ( 0 or $180 \mathrm{deg}$ ), taken from Fig. 6. The use of the local color cue produces a suprathreshold facilitation of the luminance test threshold. 

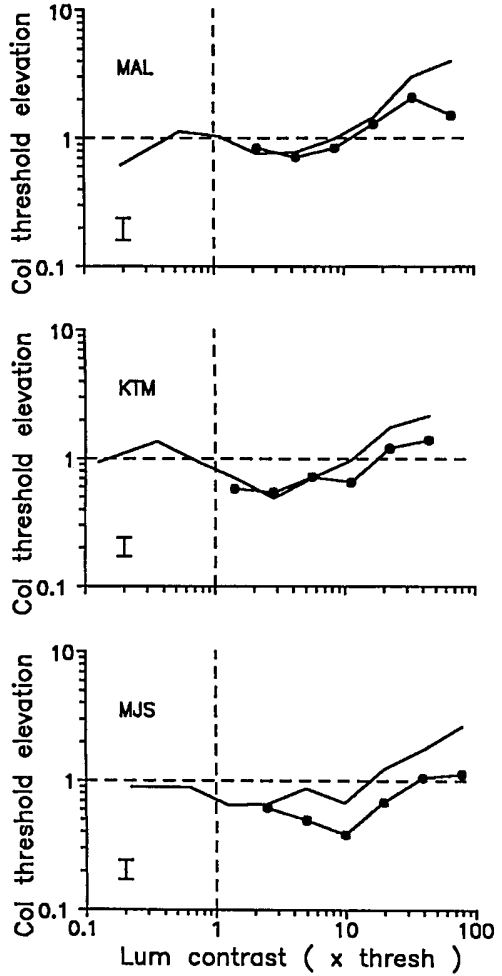

Fig. 15. Color test thresholds plotted as a function of luminance contrast for three subjects. Solid symbols, thresholds for the detection of a local color cue based on a comparison of the stimuli in the two presentation intervals (see text). Curves without symbols, random presentation of test and mask ( 0 or $180 \mathrm{deg}$ ), taken from Fig. 7. The use of the local color cue, except in the cases of subjects MAL and KTM, does not increase the suprathreshold facilitation of the color test threshold.

the luminance mask grating acquire an orange hue in the presence of the test color contrast. If the combination is made in the opposite phase, the yellow bars acquire a greener hue. For a fixed phase, for example, $180 \mathrm{deg}$, picking the greener of the yellow bars in the two intervals might provide a useful cue to detection of the color test stimulus.

As in the previous experiment, the phase of the testand-mask combination is 0 or $180 \mathrm{deg}$, randomly varied from trial to trial. The subject was specifically instructed to compare the yellow bar color between the two intervals and to select for the greener yellow. Since the greener yellow bars are associated with the presence of the color test in only one of the two phases (180 deg), the local color cue is not correlated with the presence of the color test stimulus. The results are shown in Fig. 15 by the symbols. For comparison, threshold for the randomized phase results (from Fig. 7) are shown by the solid curves. The data show that in two subjects the amount of suprathreshold facilitation is similar in both conditions. In the third subject the use of a chromaticity cue lowers thresholds in comparison with the detection of the color test alone. Thus the results suggest that attending to a local color cue is not, in general, a useful means of improving threshold. This is supported by the subjective impression of the task; a red-green color modulation across the bars of one grating appears to be a more salient feature than a comparison of the yellow bars in each interval. Furthermore, the results plotted in Fig. 7 have already shown that phase randomization does not eliminate the suprathreshold facilitation found in the fixed phase condition, suggesting that suprathreshold facilitation in this cross condition is not entirely explained by the use of a local color cue.

\section{DISCUSSION}

It was argued in Section 1 that the combination of the cone opponent and cone additive detection mechanisms within a single common pathway [the double-duty model of Fig. 1(a)] is tested by the degree of subthreshold summation between the two mechanisms. In our results we find a lack of subthreshold facilitation between chromatic and luminance mechanisms in both cross conditions, which is in agreement with previous studies ${ }^{9,12,13}$ except one. ${ }^{14}$ The lack of summation implies that there are distinct pathways mediating color and luminance detection. This conclusion, however, does not discount the likely role of $P$ cells in carrying combined color and luminance information at a subcortical level but implies that there has been an effective separation of color and luminance responses at a higher stage. Various methods by which the color and luminance responses of $P$ cells may be separated at the cortical level have been proposed. ${ }^{28}$

The existence of separate sites or pathways for the processing of color and luminance information does not imply that they are completely independent. This point was illustrated in Figs. 1(b) and 1(c), which show two possible types of interaction that could be revealed at suprathreshold contrast levels. Our data for the two crossed $\mathrm{TvC}$ functions clearly demonstrate that interactions occur between color and luminance contrast: both functions show suprathreshold facilitation, and both show some masking. Simply observing the shape of the crossed TvC functions, however, does not address the origin of these interactions. Moreover, as the separability model of Fig. 1(c) illustrates, interactions may be apparent despite an underlying independence of the color and luminance transduction processes. We found that the subthreshold facilitation of the luminance-luminance TvC function is unaffected by the presence of a suprathreshold color contrast mask. The dipper shape of the uncrossed luminance TvC function is unchanged, although its overall position is shifted upward and rightward, reflecting the cross-masking effect of the chromatic pedestal grating on the luminance thresholds. A similar effect is found for the uncrossed color TvC function, which shows some facilitation despite the initial elevation of thresholds by the fixed luminance pedestal grating. Overall, the separability of the color-color TvC function from luminance contrast is somewhat less convincing, because the dipper shape of this uncrossed function is flatter and broader than would usually be found in the absence of a luminance contrast pedestal. These results are contrary to those of Cole et al. ${ }^{13}$ which demonstrated a loss of the dipper shape of the uncrossed TvC functions in the presence of a pedestal of the other contrast. However, there are some differences in the two sets of conditions used. The results of Cole et $a l$. were obtained with pedestals of a lower fixed contrast and with a fixed phase of test and mask, as opposed to the randomized phase conditions and higher contrast 
pedestals that we used. Their conditions are optimal for eliciting suprathreshold facilitation of the test stimulus, and this may account for why no further facilitation was found when the uncrossed (same-on-same) contrast increment thresholds were measured.

The results of these experiments suggest two things. First, the results are not compatible with models that propose that an additive combination of color and luminance contrast controls test threshold. They therefore suggest that the color and luminance transduction processes at low to medium contrast have no cross sensitivity. This interpretation is independent of whether the uncrossed facilitation may be accounted for by nonlinear (accelerating) transduction processes ${ }^{30-32}$ or is due to uncertainty. ${ }^{33}$ If the suprathreshold facilitation found in the two cross conditions cannot be accounted for by a cross sensitivity between color and luminance mechanisms, other explanations for it are required.

Second, the observation that the dipper shape of the uncrossed (same-on-same) TvC functions can occur despite the fact that the test threshold is already elevated by cross masking suggests that the uncrossed facilitation and cross masking are the results of different underlying processes. In particular, it rules out an explanation for the cross masking in terms of a compressive nonlinearity in the transduction process, because such an explanation could not account for the coexistence of masking and facilitation found in our results. Alternative explanations for masking that involve a contrast gain control are available. ${ }^{34,35}$ In this case our results would suggest that a response scaling operates between color and luminance pathways. Thus it may be that masking, mediated by a divisive response scaling, displays a cross interaction between color and luminance pathways at high contrasts, whereas channel transduction for luminance and color contrast is independent. Although it is lower in magnitude, the cross masking has a similar bandpass spatial tuning to that of the uncrossed masking of the color or luminance TvC functions, ${ }^{19,25}$ suggesting that the two types of masking may have a similar origin. The cross masking also resembles the bandwidth for gain control that is assumed to be responsible for masking in spatial pattern discrimination. ${ }^{36}$ If it is assumed, however, that adaptation and masking work by similar response scaling mechanisms, ${ }^{34}$ some cross adaptation between color and luminance contrast would also be expected. In general, no cross adaptation between the color and luminance mechanisms has been found, ${ }^{15,16}$ although small effects are apparent at very high adapting contrasts.

As pointed out above, if a cross sensitivity between color and luminance pathways cannot account for the suprathreshold facilitation, other explanations must be sought. We have found that the suprathreshold facilitations in the two cross conditions have complex characteristics that also show differences between the two conditions. The enhancement of luminance contrast detection by a suprathreshold color contrast can be accounted for by a local color cue that is available to the subject in the two-alternative forced-choice paradigm. This reflects a pitfall in the use of suprathreshold discrimination experiments: at suprathreshold contrasts, local features of the test-plus-mask combination may emerge that can be learned to aid in detection of the test. Randomizing the relative phase of the test and mask from trial to trial is one means of removing these cues, and in this case (luminance detection in the presence of color contrast) largely removes the suprathreshold facilitation. A similar loss of local cues is also likely to account for the absence of facilitation when test and mask differ in spatial frequency. Threshold elevation with phase randomization has also been reported for masking experiments that use complex luminance stimuli ${ }^{37}$ and may also be accounted for by the disruption of local cues. The availability of local color cues to improve luminance detection thresholds provides a convenient accounting for the differences in the suprathreshold facilitation between our data and those of Switkes et al. ${ }^{12}$ The presence of suprathreshold facilitation in our data and its absence in the data of Switkes et $a l .{ }^{12}$ may be explained by whether the individual subjects have learned to attend to local cues in the stimuli.

In the other cross condition, suprathreshold facilitation, described here and elsewhere, ${ }^{12,13,20}$ reflects the enhancement of color detection by luminance contrast. The improvement of wavelength discrimination that is sometimes observed in the presence of small luminance contrast ${ }^{38-40}$ probably reflects the same effect. Another similar effect, known as the gap effect, has also been reported for $\mathrm{S}$ cones. ${ }^{41,42}$ The enhancement of color detection by luminance contrast appears to be a higher-order effect because a variety of spatial cues, such as thin lines around the test area, can produce the facilitation. ${ }^{13}$ This aspect is compatible with our finding that this facilitation shows no spatial tuning. It has been suggested ${ }^{13,20}$ that the spatial demarcation of a test spot area from the surround allows the subject to make a more sensitive comparison of the color of the test-plus-mask combination with the mean chromaticity of the adapting background. However, our results show that a phase randomization of test and mask stimuli with as many as 16 phases fails to remove the facilitation. This suggests that, at least for sine-wave stimuli, a between-interval color cue is not involved. The explanation for this suprathreshold facilitation thus remains something of a mystery.

Overall, our results are compatible with the existence of independent color and luminance pathways at the cortical level. Our results suggest that the transduction of luminance contrast is independent from color contrast. The independence of color contrast transduction from luminance contrast is also suggested but remains to be firmly established. Finally, there is other support in the literature for the existence of distinct color and luminance pathways that mediate higher tasks. There is evidence for an independent contribution of color and luminance contrast to reading speed ${ }^{43}$ and for the involvement of separate color and luminance processes in contour detection. ${ }^{\mathbf{4 4}}$ These findings suggest that spatial representation of color and luminance contrast remain distinct at higher levels of cortical processing.

\section{APPENDIX A}

It was pointed out in Section 2 that the selection of the chromatic and luminance stimuli is necessarily based on 
two main assumptions. The purpose of this appendix is to explore whether the relaxation of these assumptions could account for the results that we find. In short, can any of the color-luminance interactions be accounted for by the activation of more than one visual mechanism by the stimuli? The cone contrast space of Fig. 2 is used to describe the stimuli and detection mechanisms. Cone quantal catches are derived from the Smith-Pokorny cone fundamentals ${ }^{45}$ and the calibrated spectral irradiances of the monitor's three phosphors. The use of this space assumes that it is relevant to represent the quantal catches of each cone type relative to its own mean and that the detection mechanisms rely on a linear combination of the responses of these cone types. Both assumptions are supported by the literature ${ }^{4,5}$ and allow stimuli and mechanisms to be represented by vectors within the cone contrast space of Fig. 2. In what follows, $\mathbf{M}$ represents the vector directions of the detection mechanisms. The type of mechanism is defined by the subscripts $\mathrm{rg}$, lum, or by (blue-yellow). $L$ and $M$ represent the normalized cone contrast coordinates of the detection mechanisms. S represents the vector directions of the stimuli, and $l$ and $m$ represent the L- and M-cone contrasts of the stimuli, respectively.

\section{A. Direction of the L-M Chromatic Mechanism}

The first assumption is that the $\mathrm{L}-\mathrm{M}$ chromatic mechanism has equal L- and M-cone weights. Such a mechanism lies at $135 \mathrm{deg}(0.71-0.71)$ and is therefore orthogonal to the isochromatic luminance stimulus at $45 \mathrm{deg}$ (see Fig. 2). If, however, this chromatic mechanism were to deviate from this direction, the stimulus and the mechanism would no longer be orthogonal, and some cross stimulation of this mechanism by the luminance stimulus would occur. The question is whether such a tilt in the $\mathrm{L}-\mathrm{M}$ chromatic mechanism is likely to be big enough to allow the luminance stimulus to modulate it at a detectable level. As an example of why this question is important, we refer to Fig. 3(a), in which a luminance test is added to a chromatic mask. If the luminance test were in fact producing a significant modulation in the chromatic mechanism, parts of this function might reflect the contrast increment thresholds of a chromatic mechanism. Below we calculate whether the luminance stimulus is likely to produce a detectable modulation in the chromatic system. We use a worst-case example by taking the most sensitive chromatic thresholds for each subject, which occur when the chromatic mechanism is maximally facilitated at approximately one to two times threshold of a chromatic mask. We select a 5-deg tilt of the L-M chromatic vector angle from $135 \mathrm{deg}$. This is a high deviation of the chromatic mechanism, as it represents ten times its measured standard deviation (mean angle $135.3 \mathrm{deg}$, standard deviation $0.5 \mathrm{deg}$, measured for three subjects). ${ }^{8}$

The vector representing the assumed L-M chromatic mechanism is $\mathbf{M}_{\mathrm{rg}}=(-0.71,0.71)$. The isoluminant chromatic stimulus is given by $\mathbf{S}_{\mathrm{rg}}=\left(-l_{\mathrm{rg}}, m_{\mathrm{rg}}\right)$, depending on the exact red-green ratio measured for each subject. The luminance mechanism lies orthogonal to the isoluminant direction: $\mathbf{M}_{\text {lum }}=\left(L_{\text {lum }}, M_{\text {lum }}\right)$, where the vector length is normalized such that $L_{\mathrm{lum}}{ }^{2}+M_{\mathrm{lum}}{ }^{2}=1$. The homochromatic luminance stimulus is represented by a vector $\mathbf{S}_{\text {lum }}=(0.71,0.71)$.

\section{General Case}

The response of a given mechanism $\mathbf{M}=(a, b, c)$, to a given stimulus $\mathbf{S}=(x, y, z)$ is defined as the dot product of the two vectors:

$$
\mathbf{M} \cdot \mathbf{S}=|\mathbf{S}||\mathbf{M}| \cos (\tau)=a x+b y+c z,
$$

where $\tau$ is the angle between vectors $\mathbf{M}$ and $\mathbf{S}$.

\section{Specific Cases}

The response of the chromatic mechanism to a chromatic stimulus whose cone contrast values are $\left(-l_{\mathrm{rg}}, m_{\mathrm{rg}}\right)$ is given by

$$
\mathbf{M}_{\mathrm{rg}} \cdot \mathbf{S}_{\mathrm{rg}}=0.71\left(l_{\mathrm{rg}}+m_{\mathrm{rg}}\right)
$$

The response of the luminance mechanism to a homochromatic luminance stimulus whose cone contrast values are $\left(l_{\text {lum }}, m_{\text {lum }}\right)$ is

$$
\mathbf{M}_{\text {lum }} \cdot \mathbf{S}_{\text {lum }}=l_{\text {lum }} L_{\text {lum }}+m_{\text {lum }} \mathbf{M}_{\text {lum }}
$$

for any homochromatic stimulus $l_{\text {lum }}=m_{\text {lum }}$; thus

$$
\mathbf{M}_{\text {lum }} \cdot \mathbf{S}_{\text {lum }}=l_{\text {lum }}\left(L_{\text {lum }}+M_{\text {lum }}\right) .
$$

Note that the responses of the chromatic mechanism to the luminance stimulus, and of the luminance mechanism to the chromatic stimulus, are both null because these stimuli and mechanisms are orthogonal:

$$
\mathbf{M}_{\mathrm{lum}} \cdot \mathbf{S}_{\mathrm{rg}}=\mathbf{M}_{\mathrm{rg}} \cdot \mathbf{S}_{\mathrm{lum}}=0
$$

Nonorthogonality of the $L-M$ Chromatic

Mechanism and the Luminance Stimulus

If the chromatic mechanism lies not in the 135-deg direction $(-0.71,0.71)$ but in a different direction $\alpha$, it will be stimulated by the luminance stimulus. The vector direction for the tilted chromatic mechanism $\alpha$ has the coordinates

$$
\mathbf{M}_{\mathrm{rg}}=(-x, y)
$$

where $|\tan (\alpha)|=y / x$.

Thus the response of the tilted chromatic mechanism to a luminance grating $\left(l_{\text {lum }}, m_{\text {lum }}\right)$ is no longer null but is given by

$$
\mathbf{M}_{\mathrm{rg}} \cdot \mathbf{S}_{\mathrm{lum}}=l_{\mathrm{lum}}(-x+y)
$$

Let the response of the tilted chromatic mechanism to the chromatic stimulus at threshold $\left(-l_{\mathrm{rg}}, m_{\mathrm{rg}}\right)$ be given by

$$
\mathbf{M}_{\mathrm{rg}} \cdot \mathbf{S}_{\mathrm{rg}}=l_{\mathrm{rg}} x+m_{\mathrm{rg}} y .
$$

We wish to calculate the cone contrast of the luminance grating $\left(l_{\text {lum }}=m_{\text {lum }}\right)$ necessary to produce a detectable modulation in the tilted chromatic mechanism. We equate the threshold response of the chromatic mecha- 
$\operatorname{nism}\left(\mathbf{M}_{\mathrm{rg}} \cdot \mathbf{S}_{\mathrm{rg}}\right)$ to the response of the chromatic mechanism to a luminance grating $\left(\mathbf{M}_{\mathrm{rg}}, \mathbf{S}_{\mathrm{lum}}\right)$ :

$$
l_{\mathrm{lum}}(-x+y)=l_{\mathrm{rg}} x+m_{\mathrm{rg}} y .
$$

Thus the value of the cone contrast of the luminance stimulus that produces a detectable stimulation of the chromatic mechanisms is given by

$$
l_{\text {lum }}=\frac{l_{\mathrm{rg}}|\tan (\alpha)|+m_{\mathrm{rg}}}{|\tan (\alpha)|-1} .
$$

Values of $l_{\mathrm{rg}}$ and $m_{\mathrm{rg}}$ were obtained from each subject and represent the most sensitive detection thresholds for the isoluminant red-green grating, obtained at maximum facilitation. For MAL, KTM, and MJS, the $l_{\mathrm{rg}}$ and $m_{\mathrm{rg}}$ values are $-0.00036,0.0018 ;-0.00055,0.0020 ;-0.00075$, 0.0018 , respectively. When the chromatic mechanism is tilted toward the $M$ axis by $5 \mathrm{deg}$ ( $\alpha=130 \mathrm{deg}$ ), a screen luminance contrast of 0.015 , averaged over the three subjects, is required for production or a detectable modulation in the deviated L-M color mechanism.

This luminance contrast $(0.015)$ is well above the measured luminance threshold over the relevant color mask values [see Fig. 3(a)]. Thus the luminance mechanism, rather than a deviated color mechanism, will determine detection threshold. In reality, the effective cross stimulation of the chromatic mechanism will be much less than we have assumed, because the chromatic vector is unlikely to deviate by as much as ten standard deviations, and the color threshold will generally be higher than the most sensitive, facilitated threshold that we have used. Thus the assumption that the chromatic mechanism is effectively orthogonal to the luminance stimulus is valid.

\section{B. Detection of Luminance or Chromatic Stimuli} by the $S /(L+M)$ Mechanism

The second assumption that we make is that the $S /(L+$ $M$ ) mechanism does not detect either the luminance or the red-green chromatic stimuli. This mechanism may potentially be excited by the yellow luminance grating or by the isoluminance red-green grating. The amount of the cross stimulation can be calculated with certain assumptions. The direction of the $S /(L+M)$ vector needs to be known. On the basis of the available literature, ${ }^{8}$ we have taken L-, M- and S-cone weightings of $-0.73,-0.11$ and 0.67 , respectively, although it should be pointed out that these estimates are highly variable. The representation of this vector in Fig. 2 has not been plotted but would be rising above and falling below the page in the third and the first quadrants, respectively. Neither the luminance nor the red-green chromatic stimuli are orthogonal to this $S /(L+M)$ mechanism. [Nor would they be orthogonal to a conventionally weighted $S /(L+M)$ mechanism of $-0.41,-0.41$, and 0.82 ].

The stimulation of the blue-yellow chromatic mechanism by luminance and red-green isoluminant stimuli is given by

$$
\begin{aligned}
\left|\mathbf{M}_{\text {by }} \cdot \mathbf{S}_{\text {lum }}\right| & =0.84 l_{\text {lum }}, \\
\left|\mathbf{M}_{\text {by }} \cdot \mathbf{S}_{\mathrm{rg}}\right| & =\left|0.73 l_{\mathrm{rg}}-0.11 m_{\mathrm{rg}}\right| .
\end{aligned}
$$

We compare the stimulation of the luminance mechanism and the stimulation of the $S /(L+M)$ mechanism by use of a luminance stimulus of a fixed contrast (unit vector length). Averaged over the three subjects, the luminance stimulus stimulates the luminance mechanism 1.5 times more than it does the $S /(L+M)$ mechanism. The effectiveness of this difference, however, depends on the relative thresholds of the two mechanisms at the spatial frequency that we have used ( 1 cycle/deg), for which data are not available. One cycle/deg, however, is at the peak of the luminance contrast-sensitivity function but on the declining part of the color contrast-sensitivity function, suggesting that the luminance mechanism is likely to be more sensitive than the $S /(L+M)$ mechanism and will determine detection threshold for the yellow-black gratings. Furthermore we note that, even at the highest contrasts, the black bars of the yellow-black grating never acquire a bluish hue. Thus the assumption that the $S /(L+M)$ mechanism does not detect the luminance stimulus appears valid.

The red-green chromatic stimulus stimulates the L-M chromatic mechanism 13.5 times more than it does the $S /(L+M)$ mechanism. Since the L-M mechanism is very sensitive in terms of cone contrasts, it is highly unlikely that the $S /(L+M)$ mechanism will contribute to detection of the red-green chromatic stimulus.

We conclude from this appendix that luminance and chromatic stimuli used in the experiments are effectively cardinal and that the results cannot be accounted for by the activation of more than one visual mechanism by these stimuli.

\section{ACKNOWLEDGMENTS}

We thank M. J. Sankeralli for acting as a subject and F. A. A. Kingdom for acting as a subject for Fig. 3 . We thank J. C. Boulton, F. A. A. Kingdom, and W. H. McIlhagga for helpful discussion. The work was supported by Canadian Medical Research Council grant MT 10819.

\section{REFERENCES}

1. H. G. Sperling and R. S. Harwerth, "Red-green cone interactions in the increment threshold spectral sensitivity of primates," Science 172, 180-184 (1971).

2. P. E. King-Smith and D. Carden, "Luminance and opponent colour contributions to visual detection and adaptation and to spatial and temporal integration," J. Opt. Soc. Am. 66, 709-717 (1976).

3. C. Noorlander, M. J. G. Heuts, and J. J. Koenderink, "Sensitivity to spatiotemporal combined luminance and chromaticity contrast," J. Opt. Soc. Am. 71, 453-459 (1981).

4. C. F. Stomeyer, R. E. Kronauer, and G. R. Cole, "Adaptive mechanism controlling sensitivity to red-green chromatic flashes," in Colour Vision: Physiology and Psychophysics, J. D. Mollon and L. T. Sharpe, eds. (Academic, London, 1983), pp. 313-330.

5. C. F. Stomeyer, G. R. Cole and R. E. Kronauer, "Second-site adaptation in the red-green chromatic pathways," Vision Res. 25, 219-237 (1985).

6. C. F. Stomeyer, G. R. Cole, and R. E. Kroneuer, "Chromatic supression of cone inputs to the luminance flicker mechanism," Vision Res. 27, 1113-1137 (1987).

7. P. E. King-Smith, A. J. Vingrys, and S. C. Benes, "Visual thresholds measured with color video monitors," Color Res. Appl. 12, 73-80 (1987).

8. G. R. Cole, T. Hine, and W. H. McIlhagga, "Detection mechanisms in L-, M-, and S-cone contrast space," J. Opt. Soc. Am. A 10, 38-51 (1993). 
9. A. Chaparro, C. F. Stromeyer, R. E. Kroneauer, and R. T. Eskew, Jr., "Separable red-green and luminance detectors for small flashes," Vision Res. 34, 751-762 (1994).

10. K. T. Mullen and J. J. Kulikowski, "Wavelength discrimination at detection threshold," J. Opt. Soc. Am. A 7, 733-742 (1990).

11. J. Krauskopf, D. R. Williams, M. B. Mandler, and A. M. Brown, "Higher order color mechanisms," Vision Res. 26, 23-31 (1986).

12. E. Switkes, A. Bradley, and K. K. De Valois, "Contrast dependence and mechanisms of masking interactions among chromatic and luminance gratings," J. Opt. Soc. Am. A 5, 1149-1162 (1988).

13. G. R. Cole, C. F. Stromeyer, and R. E. Kronauer, "Visual interactions with luminance and chromatic stimuli," J. Opt. Soc. Am. A 7, 128-140 (1990).

14. M. Gur and V. Akri, "Isoluminant stimuli may not expose the full contribution of color to visual functioning: spatial contrast sensitivity measurements indicate interaction between color and luminance processing," Vision Res. 32, 1253-1262 (1992).

15. J. Krauskopf, D. R. Williams, and D. W. Heeley, "Cardinal directions of colour space," Vision Res. 22, 1123-1131 (1982).

16. A. Bradley, E. Switkes, and K. K. De Valois, "Orientation and spatial frequency selectivity of adaptation to color and luminance gratings," Vision Res. 28, 841-856 (1988).

17. M. A. Webster and J. D. Mollon, "Changes in colour appearance following post receptoral adaptation," Nature (London) 349, 235-238 (1991).

18. K. R. Gegenfurtner and D. C. Kiper, "Contrast detection in luminance and chromatic noise," J. Opt. Soc. Am. A 9 , 1880-1888 (1992).

19. K. K. De Valois and E. Switkes, "Simultaneous masking interactions between chromatic luminance gratings," J. Opt. Soc. Am. 73, 11-18 (1983).

20. R. T. Eskew, C. F. Stromeyer, C. J. Picotte, and R. E. Kronauer, "Detection uncertainty and the facilitation of chromatic detection by luminance contours," J. Opt. Soc. Am. A 8, 394-403 (1991).

21. K. T. Mullen, "The contrast sensitivity of human colour vision to red/green and blue/yellow chromatic gratings," J. Physiol. 359, 381-400 (1985).

22. J. D. Moreland, "Spectral sensitivity measured by motion photometry," in Documenta Ophthalmologia Proceedings Series 33, G. Verriest, ed. (Junk, The Hague, 1982), pp. 61-66.

23. P. Cavanagh, C. W. Tyler, and O. E. Favreau, "Perceived velocity of moving chromatic gratings," J. Opt. Soc. Am. A 1, 893-899 (1984).

24. K. T. Mullen and J. C. Boulton, "Absence of smooth motion perception in colour vision," Vision Res. 32, 483-488 (1992).

25. M. A. Losada and K. T. Mullen, "The spatial tuning of chromatic mechanism identified by simultaneous masking," Vision Res. 34, 331-341 (1994).

26. G. E. Legge and J. M. Foley, "Contrast making in human vision," J. Opt. Soc. Am. 70, 1458-1471 (1980).

27. A. Bradley, X. Zhang, and L. Thibos, "Failures of isolu- minance by ocular chromatic aberration," Appl. Opt. 31, 3657-3667 (1992)

28. F. A. A. Kingdom and K. T. Mullen, "Separating colour and luminance in the brain," Spatial Vision (to be published).

29. K. T. Mullen, M. A. Losada and F. A. A. Kingdom, "Inherent stimulus non-linearities in the combination of color and luminance contrast," Invest. Ophthalmol. Vis. Sci. Suppl. 33, 699 (1992).

30. J. Nachmias and R. V. Sansbury, "Granting contrast: discrimination may be better than detection," Vision Res. 14, 1039-1042 (1974)

31. C. F. Stromeyer and S. Klein, "Spatial frequency channels in human vision as asymmetric (edge) mechanisms," Vision Res. 14, 1409-1419 (1974).

32. J. M. Foley and G. E. Legge, "Contrast detection and near threshold discrimination in human vision," Vision Res. 21, 1041-1053 (1981).

33. D. G. Pelli, "Uncertainty explains many aspects of visual contrast detection and discrimination," J. Opt. Soc. Am. A 2, 1508-1531 (1985).

34. J. Ross and H. D. Speed, "Contrast adaptation and contrast masking in human vision," Proc. R. Soc. London Ser. B 246, 61-70 (1991)

35. J. M. Foley, Pattern masking phenomena require a new model of human pattern vision mechanisms," Invest. Ophthalmol. Vis. Sci 34, 819 (1993).

36. L. A. Olzak and J. P. Thomas, "Normalizing and summing pools in complex frequency discriminations," Invest. Ophthalmol. Vis. Sci. 34, 818 (1993).

37. J. Nachmias, "Contrast modulated maskers: test of a late non-linearity hypothesis," Vision Res. 29, 137-142 (1989).

38. R. Hilz and C. R. Cavonius, "Wavelength discrimination measured with square-wave gratings," J. Opt. Soc. Am. 60, 273-277 (1970).

39. R. Hilz, G. Huppmann, and C. R. Cavonius, "Influence of luminance contrast on hue discrimination," J. Opt. Soc. Am. 64, 763-766 (1974).

40. A. E. Elsner, J. Pokorny, and S. A. Burns, "Chromaticity discrimination: effects of luminance contrast and spatial frequency," J. Opt. Soc. Am. A 3, 916-919 (1986).

41. R. M. Boynton, M. M. Hayhoe, and D. I. A. Macleod, "The gap effect: chromatic and achromatic visual discrimination as affected by field separation," Opt. Acta 24, 159-177 (1977).

42. R. T. Eskew "The gap effect revisited: slow changes in chromatic sensitivity as affected by luminance and chromatic borders," Vision Res. 29, 717-729 (1989).

43. G. E. Legge, D. H. Parish, A. Luebker, and L. H. Wurm, "Psychophysics of reading. XI. Comparing color contrast and luminance contrast," J. Opt. Soc. Am. A 7, 2002-2010 (1990).

44. W. H. McIlhagga and K. T. Mullen, "Contour detection with chromatic and luminance contrast," Vision Res. (to be published).

45. V. C. Smith and J. Pokorny, "Spectral sensitivity of the foveal cone pigments between 400 and $500 \mathrm{~nm}$," Vision Res. 15, 161-171 (1975). 